\title{
SCIENTIFIC REPORTS

\section{Variations in microbial community structure and functional gene expression in bio-treatment processes with odorous pollutants}

\author{
Weidong Li $\mathbb{1}^{1}$, Jianguo $\mathrm{Ni}^{2}$, Shaoqin $\mathrm{Cai}^{3,4}$, Ying Liu ${ }^{3}$, Chenjia Shen ${ }^{3}$, Huayun Yang ${ }^{1}$, \\ Yuquan Chen ${ }^{3}$, Jia Tao ${ }^{3}$, Yunfeng Yu ${ }^{3}$ \& Qi Liu ${ }^{1,3^{*}}$
}

Engineered microbial ecosystems in biofilters have been widely applied to treat odorous gases from industrial emissions. Variations in microbial community structure and function associated with the removal of odorous gases by biofilters are largely unknown. This study performed a metagenomic analysis to discover shifts in microbial community structures in a commercial scale biofilter after treating odorous gas. Our study identified 175,675 functional genes assigned into 43 functional KEGG pathways. Based on the unigene sequences, there were significant changes in microbial community structures in the biofilter after treating odorous gas. The dominant genera were Thiobacillus and Oceanicaulis before the treatment, and were Acidithiobacillus and Ferroplasma after the treatment. A clustering analysis showed that the number of down-regulated microbes exceeded the number of up-regulated microbes, suggesting that odorous gas treatment reduced in microbial community structures. A differential expression analysis identified 29,975 up- and 452,599 down-regulated genes. An enrichment analysis showed 17 classic types of xenobiotic biodegradation pathways. The results identified 16 and 15 genes involved in ammonia and sulfite metabolism, respectively; an analysis of their relative abundance identified several up-regulated genes, which may be efficient genes involved in removing odorous gases. The data provided in this study demonstrate the changes in microbial communities and help identify the dominant microflora and genes that play key roles in treating odorous gases.

Odorous gases from industrial emissions significantly contribute to haze pollution and photochemical contamination in China ${ }^{1}$. Odorous pollutants, including major and typical outdoor air pollutants, threaten personal health and deteriorate public welfare ${ }^{2}$. Odors generally consist of ammonia and sulfur compounds; and complex odors have detrimental effects on ecological and environmental functions $s^{3,4}$. Thus, reducing odorous emission sources is an effective strategy for controlling photochemical air pollution ${ }^{5}$.

The national emission standards of odorous has be updated recently, requiring stricter emission standards (DB33-2146-2018) than before. Many treatment methods, including physical, chemical, and biological technologies, have been developed to limit odorous gas emissions ${ }^{6}$. Physical-chemical methods effectively treat odorous gases; however, their application depend on having high concentrations, come at a high cost, and create secondary pollutions ${ }^{7}$. Several biotechnologies, including biofilters, bioscrubbers, and biotrickling filters, are currently considered the most promising and environment-friendly approaches for treating low concentrations of odorous gases $^{8}$. Due to differences in the microbial community and liquid states, different bioreactors are used to treat different kinds of pollutants ${ }^{2}$. Biofiltration has become a viable and economic technology to treat industrial emissions containing low concentrations of odorous gases ${ }^{9}$.

Degrading pollutants into smaller, less harmful molecules depends on the capacity of a highly effective microbial community ${ }^{10}$. Recently, there have been more studies on the structural and functional responses of microbial

${ }^{1}$ College of Qianjiang, Hangzhou Normal University, Hangzhou, 310036, Zhejiang, People's Republic of China. ${ }^{2}$ Hangzhou Ecological Environment Bureau of Xiaoshan Branch, Hangzhou, 311201, Zhejiang, People's Republic of China. ${ }^{3}$ College of Life and Environmental Science, Hangzhou Normal University, Hangzhou, 310036, Zhejiang, People's Republic of China. ${ }^{4}$ College of Environment, Zhejiang University of Technology, Hangzhou, 310014, Zhejiang, People's Republic of China. *email: qiliu@hznu.edu.cn 
communities when treating odorous gases. For example, reactors supplied with a gaseous effluent resulted in the simplification of the structure of a bacterial community and the appearance of a dominant microflora ${ }^{11}$. Another study investigated the correlations between odor and microbial composition during food waste composting ${ }^{12}$. Analyzing microbial communities is a shortcut to screen high-efficiency strain and optimize a biofiltration system.

Microbial communities play essential roles in engineered microbial ecosystems ${ }^{13}$. Research has identified a number of waste gas catabolic microbial strains ${ }^{13}$. For example, studies have investigated the role of Sphingomonas sp. "D3K1" in degrading ethylbenzene; Thiomonas sp. "WZW" in degrading carbon disulfide; and Acidithiobacillus thiooxidans in degrading hydrogen sulfide ${ }^{14-16}$. High-throughput sequencing has shown to be effective in revealing variations in microbial communities ${ }^{17}$. To uncover the microbial characteristics in bioreactors, previous studies have also developed new metagenomic sequencing techniques ${ }^{18,19}$. However, how complex gases containing $\mathrm{H}_{2} \mathrm{~S}$ and $\mathrm{NH}_{3}$ effect the microbial community structure in a biofilter system are largely unknown.

This study applied high-throughput metagenomics sequencing to investigate how microbial compositions and gene transcript profiles respond to the biodegradation of odorous gases in a biofilter system. Several target strains and functional genes will be used for improving the efficiency of biofilter. The data provided by this study demonstrates the changes in the microbial communities and helps identify the dominant microflora that play key roles in treating odorous gases.

\section{Materials and Methods}

Materials and sampling. Mixed media, containing activated carbon, wheat bran, and sawdust (1:1:2), was used to biodegrade the odorous gases. Sample materials were enriched using activated sludge from a waste gas treatment plant (Wenzhou, China); there was a 3-week acclimation period with continuous clean air (Before Treatment, BT), followed by a week period of continuous odor-contaminated airflow (After Treatment, AT). The main components of odorous gases are $\mathrm{H}_{2} \mathrm{~S}$ and $\mathrm{NH}_{3}$. The initial concentrations of $\mathrm{H}_{2} \mathrm{~S}$ and $\mathrm{NH}_{3}$ are $35.3 \mathrm{mg} \cdot \mathrm{m}^{-3}$ and $16.1 \mathrm{mg} . \mathrm{m}^{-3}$, respectively. The emission rate of $\mathrm{H}_{2} \mathrm{~S}$ and $\mathrm{NH}_{3}$ are $0.26 \mathrm{~kg} . \mathrm{h}^{-1}$ and $0.26 \mathrm{~kg} . \mathrm{h}^{-1}$, respectively. The contaminated air passed through the media in an up-flow direction. Figure la shows the details of the biofilter construction.

Chemical analytical methods. The odor was determined using the classical a sensory test method termed the triangle-odor-bag method ${ }^{20}$. In detail, five sniffers were employed as a panel group. During the determination, two vacuum bags were filled with gases from the biofilter's inlets and outlets using a vacuum pump. If the sniffer identified the smelly bags correctly, the smelly bags were diluted and sniffed again. The experiment was stopped until the odour concentration of the diluted sample was lower than the olfactory threshold of the sniffer. Each sample was measured by five sniffers at the same time. Finally, the odour concentration was obtained according to the individual threshold of the sniffer and the average threshold of the sniffing group. The odor index was defined using a series of formula described by Noguchi's work ${ }^{21}$. The $\mathrm{NH}_{3}$ concentrations were measured using the Nessler's reagent colorimetry method ${ }^{22}$. The $\mathrm{H}_{2} \mathrm{~S}$ concentrations were measured using the methylene blue spectrophometry method (GB/T11742-89, China).

DNA isolation. The microbial samples were extracted from the media of biofilter. For the BT group, the sample was extracted after a 3-week acclimation period with continuous clean air. After a 3-week acclimation, the sample of AT group was extracted after a week period of continuous odor-contaminated airflow. Total DNAs were isolated from $10.0 \mathrm{~mL}$ sample aliquots using a DNA kit (D4015-02, Omega, Inc., USA) following the kit's procedures. The reagent was added to obtain trace DNA from each sample, and was effectively used to prepare the DNA of most microbes. Control samples consisting of blank swabs were treated through the DNA kit to confirm they contain no DNA amplifications. DNAs were eluted in a $50 \mu \mathrm{L}$ elution buffer according to the manufacturer's method (QIAGEN). The samples were stored at $-80^{\circ} \mathrm{C}$ until used by LC-BIO TECHNOLOGIES (Hangzhou) CO., LTD. China.

Preparation of DNA libraries. Two sequencing libraries were developed to refer to the digested sludge sample before treatment (BT) and after treatment (AT). These were constructed using $5 \mu \mathrm{g}$ of genomic DNA. Preparation of DNA libraries were performed according to the previous published work ${ }^{23}$. The DNA libraries were constructed using the Nano DNA Library Preparation Kit (FC-121-4001). The DNA was fragmented using NEB dsDNA Fragmentase during a $30-\mathrm{min}$ incubation period at $37^{\circ} \mathrm{C}$. Construction of DNA library start with DNA fragments. Blunt-end fragments were produced using a fill-in reactions. Fragment sizes were selected using sample purification beads. A-bases were added to the blunt ends of strands, ensuring them to be ligated to the adapters. Each adapter contained a T-base overhang for ligating the adapter to the A-tailed DNA fragments. The adapter contains the complement of sequencing primer hybridization sites for single, paired-end, and indexed reads. Single- or dual-index adapters were added to the DNA fragments and the final products were amplified using the following PCR conditions: an initial denaturation at $95^{\circ} \mathrm{C}$ for $3 \mathrm{~min} ; 8$ cycles of denaturation at $98^{\circ} \mathrm{C}$ for $15 \mathrm{~s}$, annealing at $60^{\circ} \mathrm{C}$ for $15 \mathrm{~s}$, and extension at $72^{\circ} \mathrm{C}$ for $30 \mathrm{~s}$; and then final extension at $72^{\circ} \mathrm{C}$ for $5 \mathrm{~min}$.

Illumina sequencing and quality control. High-throughput sequencing was conducted on a HiSeq4000 platform, with the sequencing mode set at PE150. Raw sequences were treated to get valid reads. Adapters were removed from raw reads using software Cutadapt v1.9. Then, low quality reads were processed using Fqtrim v0.94 with a sliding-window algorithm. The raw sequence data has been submitted to the NCBI as a BioProject with accession number PRJNA522625. 


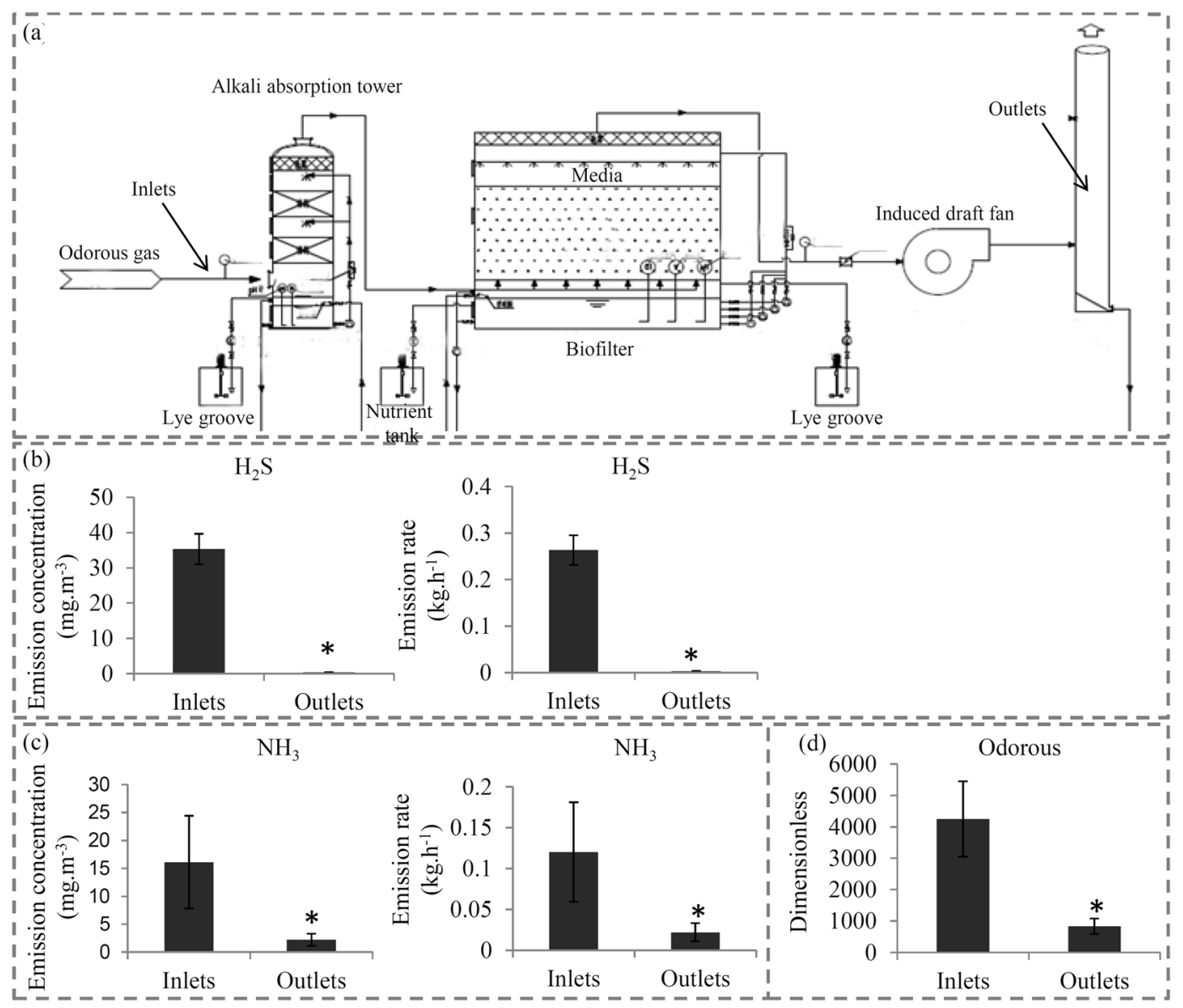

Figure 1. Removal performance for odorous gases of our biofilter construction. (a) A sketch map showed the details of the biofilter construction in our study. (b) The emission concentrations and emission rates of $\mathrm{H} 2 \mathrm{~S}$ in the inlet and outlet of the biofilter construction. (c) The emission concentrations and emission rates of NH3 in the inlet and outlet of the biofilter construction. (d) The dimensionless of odorous gases in the inlet and outlet of the biofilter construction. The significant variations ( $\mathrm{p}<0.05$ between the inlet and outlet were indicated by "*”. Error bars represent mean $\pm S D(n=3)$.

De novo assembly and unigene analysis. De novo assembly and unigene analysis were performed according to the previous published work ${ }^{24}$. The clean reads were de novo assembled to produce the metagenome using software SPAdes v3.10.0. All coding sequences (CDS) of the metagenomic contigs were predicted using software MetaGeneMark v3.26. The CDSs of all sample groups were clustered by software CD-HIT v4.6.1 to get unigenes. The abundance of unigene in each sample were evaluated by Transcripts Per Million (TPM) based on the number of aligned reads using bowtie $2 \mathrm{v}$ 2.2.0. The lowest common ancestor taxonomy of unigenes were got by aligning unigene sequences against the NR, GO, KEGG, CAZy, CARD and PHI databases by software DIAMOND v. 0.7.12 with default parameters. Based on the taxonomic and functional annotation of unigenes, along with the abundance profile of unigenes, the differential analysis were conducted at each taxonomic or functional or gene-wise level, using the Kruskal-Wallis test.

Analysis of taxonomic profile. The unigenes were searched against the Nr_meta database (blastp, evalue $<1 \mathrm{e}^{-5}$ ) using the DIAMOND software, and the results with evalue $<1 \mathrm{e}^{-10}$ were selected for species classification. Applying the NCBI Taxonomy system, each classification level of the sequence was obtained using a LCA algorithm using MEGAN software, including the SuperKingdom, Phylum, Class, Order, and Family levels. The diversity of microbial communities in the BT and AT samples was evaluated using the Chaol richness index and Shannon diversity index from the unigene data at different classification levels ${ }^{25}$.

Statistical analysis. Three parallel experiments were carried out for sequencing. All data on the diversity indexes, the relative abundances of unigenes, and the $\mathrm{H}_{2} \mathrm{~S}$ and $\mathrm{NH}_{3}$ levels were processed using SPSS 18.0. One-way analysis of variance (ANOVA) was applied to analyze the differences between the BT and AT sample groups. Wilcoxon tests were conducted to detect differences in microbial community structures between two groups. The $P$ value was produced by the false discovery rate (FDR) analysis and adjusted using the Benjamini and Hochberg's method ${ }^{26}$. A significant difference was indicated by a probability value $(P)$ less than 0.05 . 


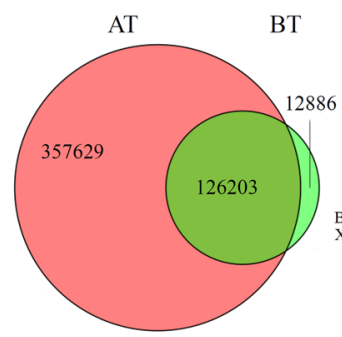

(b)

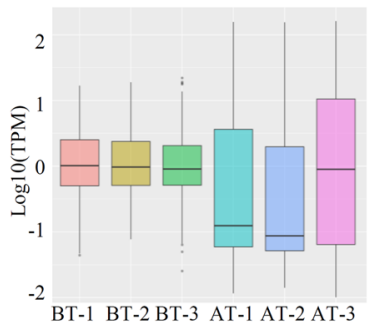

Development 0

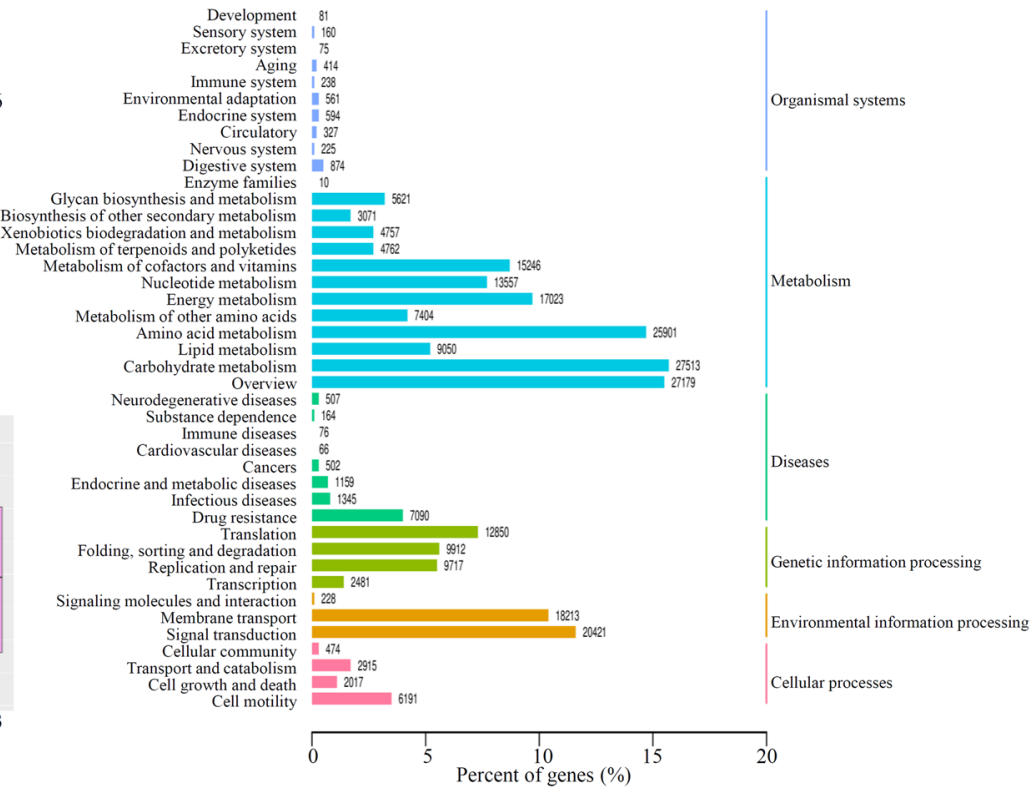

KEGG pathway classification

Figure 2. Overview of the metagenomes. (a) The numbers of unigene identified in the BT and AT sample groups are shown in a Venn diagram. Red indicated the unigenes in the AT sample group and green indicated the unigenes in the BT sample group. (b) The box-plot analysis showed that ranges of the expression abundances of unigenes from both of the BT and AT sample groups. (c) The KEGG functional prediction and classification of all identified unigenes.

\section{Results}

Removal performance for odorous gases. The study evaluated biofilter performance in removing complex mixture gases containing $\mathrm{H}_{2} \mathrm{~S}, \mathrm{NH}_{3}$, and other odorous gases. After treatment, the emission concentrations of $\mathrm{H}_{2} \mathrm{~S}$ decreased from $35.3 \mathrm{mg} \cdot \mathrm{m}^{-3}$ to $0.32 \mathrm{mg} \cdot \mathrm{m}^{-3}$; the emission rate of $\mathrm{H}_{2} \mathrm{~S}$ were reduced from $0.26 \mathrm{~kg} \cdot \mathrm{h}^{-1}$ to $0.0032 \mathrm{~kg} \cdot \mathrm{h}^{-1}$ (Fig. 1b). After treatment, emission concentrations of $\mathrm{NH}_{3}$ decreased from $16.1 \mathrm{mg} \cdot \mathrm{m}^{-3}$ to $2.2 \mathrm{mg}$. $\mathrm{m}^{-3}$; the emission rate of $\mathrm{NH}_{3}$ were reduced from $0.26 \mathrm{~kg} \cdot \mathrm{h}^{-1}$ to $0.0032 \mathrm{~kg} \cdot \mathrm{h}^{-1}$ (Fig. 1c). The biofilter absorbed $80.4 \%$ of odorous material as a result of the treatment (Fig. 1d).

Overview of the metagenomes. A total of $1.8 \mathrm{E}+08$ and $1.6 \mathrm{E}+08$ raw reads were obtained from the BT and AT samples, respectively. After filtering, 3.2E + 08 clean reads were produced to assemble the metagenome (Table S1). Pair-wise Pearson's correlation coefficients of the three replicates $\times$ two sample groups indicated the sequencing data had good repeatability (Fig. S1). The coding region of all assembled contigs was predicted to be 496,718 open reading frames (ORFs), with an average length of $576 \mathrm{bp}$.

Functional gene prediction and classification. In total, the study identified 126,203 common unigenes, 357,629 AT-specific unigenes, and 12,886 BT-specific unigenes (Fig. 2a). Figure S2a shows the length distribution of all predicted unigenes; Fig. S2b shows the densities of unigene expression in each sample. The box-plot analysis shows that the ranges of the expression abundances of unigenes from the AT group exceeded the ranges from the BT group (Fig. 2b).

Most unigenes were mapped to different KEGG pathways. In total, 175,675 unigenes were assigned to 43 functional KEGG pathways. In the 'organismal systems' category, the most represented KEGG pathways were the 'digestive system' (874 unigenes), 'endocrine system' (594 unigenes) and 'environmental adaptation' (561 unigenes). In the 'metabolism' category, the most represented KEGG pathways were 'carbohydrate metabolism' (27,513 unigenes), 'amino acid metabolism' (25,901 unigenes), and 'energy metabolism' (17,023 unigenes). In the 'diseases' category, the largest KEGG term was 'drug resistance' (7,090 unigenes). In the 'genetic information processing' category, most unigenes were grouped into the 'translation' (12,850 unigenes), 'folding, sorting and degradation' (9,912 unigenes), and 'replication and repair' (9,717 unigenes) terms. In the 'environmental information processing' category, most unigenes were grouped into the 'membrane transport' (18,213 unigenes) and 'signal transduction' (20,421 unigenes). In the 'cellular processes' category, the largest KEGG term was 'cell motility’ (6,191 unigenes) (Fig. 2c).

Comparative taxonomic profile of BT and AT metagenomes. To generate microbial information, 496,718 unigene sequences were used for the taxonomic and functional analyses (Table S2). Significant differences in microbial community structures were observed between the BT and AT sample groups. A clustering analysis showed that the number of down-regulated microbes exceeded the number of up-regulated microbes (Fig. 3a). 
(a)

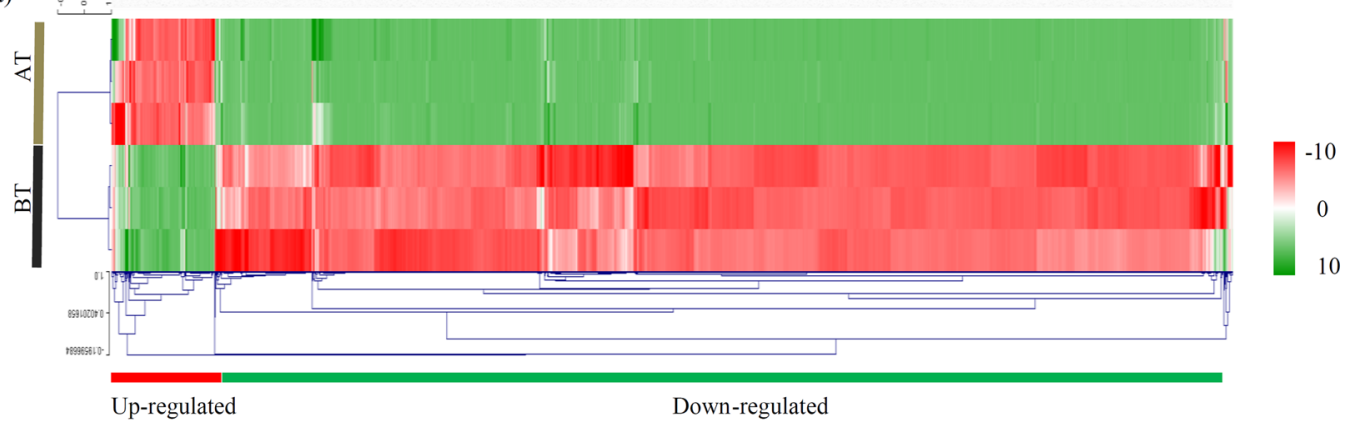

(b)
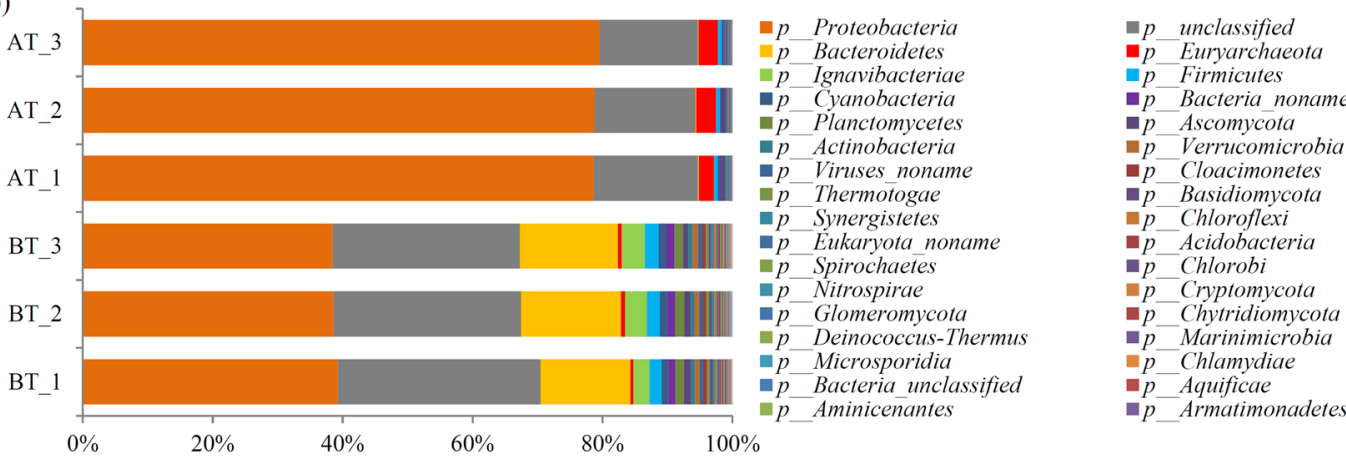

Figure 3. Comparative taxonomic profile of BT and AT metagenomes. (a) Microbial compositions for both BT and AT sample groups at genus level. Color intensity in each panel shows the relative abundances of each representative species in the BT and AT sample groups. (b) Microbial compositions for both BT and AT sample groups at phylum level. The scale unit of the heatmap is RPKM (Reads Per Kilobase per Million mapped reads). The heatmap scale ranges from -10 to +10 on a $\log _{2}$ (RPKM).

At the phylum level, the major phyla were Proteobacteria, Bacteroidetes, and Ignavibacteriae. The proportions of Proteobacteria and Euryarchaeota increased significantly, from $38.76 \%$ to $78.99 \%$ and from $0.57 \%$ to $2.77 \%$, respectively. Additionally, the proportions of Nitrospirae and Parvarchaeota were also significantly up-regulated after treatment. The proportion of other phyla significantly decreased after treatment, as follows: Bacteroidetes (from $14.77 \%$ to $0.11 \%$ ), Ignavibacteriae (from $3.15 \%$ to $0.012 \%$ ), Firmicutes ( $1.97 \%$ to $0.55 \%$ ), and Cyanobacteria ( $1.1 \%$ to $0.38 \%)$ (Fig. 3b and Table S3). The major genera were Thiobacillus $(0.51 \%)$ and Oceanicaulis $(0.27 \%)$ in the BT samples; the major genera were Acidithiobacillus (70.1\%) and Ferroplasma (2.58\%) in the AT samples. At the genus level, 7 genera were only detected in the AT samples and 254 genera were only detected in the BT samples (Table S4).

Analysis of DEGs. The study identified a large number of DEGs, including 29,975 up-regulated and 452,599 down-regulated genes (Fig. 4a and Table S5). A significance analysis of the DEGs between the AT and BT sample groups was visualized using a volcano plot (Fig. 4b). Among these DEGs, most unigenes were annotated from sequence-based homologies. For the GO classification, the top three largest GO terms in biological process were "biological process", "proteolysis", and "DNA replication." In the cellular component, the top three largest GO terms were "cellular component", "outer membrance-bounded periplasmic apace", and "cell outer membrance." In the molecular function, the three largest GO terms were "molecular function", "transporter activity", and "phosphorelay sensor kinase activity" (Fig. S3).

Enrichment analysis of the xenobiotic biodegradation pathways. All the DEGs were assigned into 387 KEGG metabolic pathways, 17 pathways of which were significantly enriched. Among these KEGG pathways, three were the most significantly enriched: 'two-component system' (map02020), 'bacterial secretion system' (map03070), and 'cationic antimicrobial peptide resistance' (map01503) (Fig. S4).

In this study, 17 classic types of xenobiotic biodegradation pathways were identified in the metagenomics datasets. Of these, the following pathways contained the largest number of up-regulated genes (Fig. 4c): 'degradation of aromatic compounds' (77 unigenes), 'benzoate degradation' (72 unigenes), 'aminobenzoate degradation' (42 unigenes), 'chloroalkane and chloroalkene degradation' (39 unigenes), and 'nitrotoluene degradation' (35 unigenes).

Expression analysis of the genes involved in nitrogen and sulfur metabolism. $\mathrm{H}_{2} \mathrm{~S}$ and $\mathrm{NH}_{3}$ are major components in odorous gases ${ }^{27}$. Our study identified a number of key genes involved in metabolizing nitrogen and sulfur; these genes play essential roles in assimilating $\mathrm{H}_{2} \mathrm{~S}$ and $\mathrm{NH}_{3}$. For the nitrogen metabolism pathway, 16 key genes were identified: nirK, niT, nirA, nirB, $n r f A$, hao, pmoA-amoA, glnA, GDH2, gluD, gltB, GLT1, 


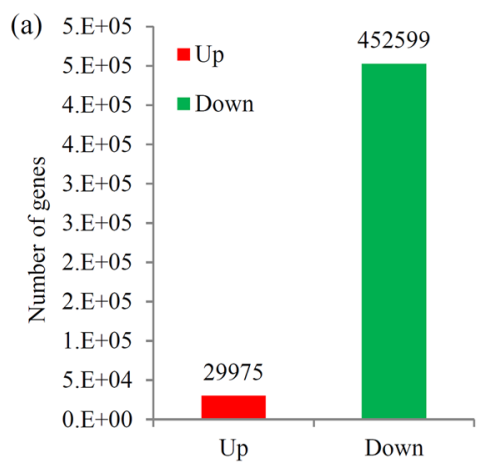

(b)

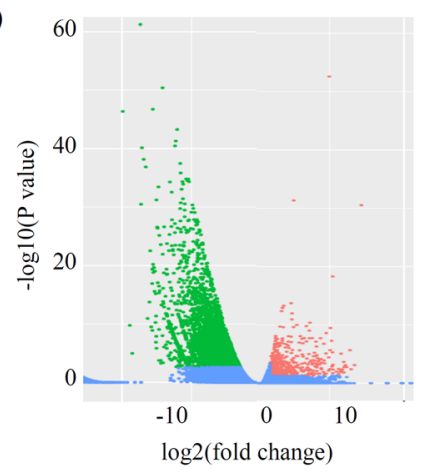

(c)

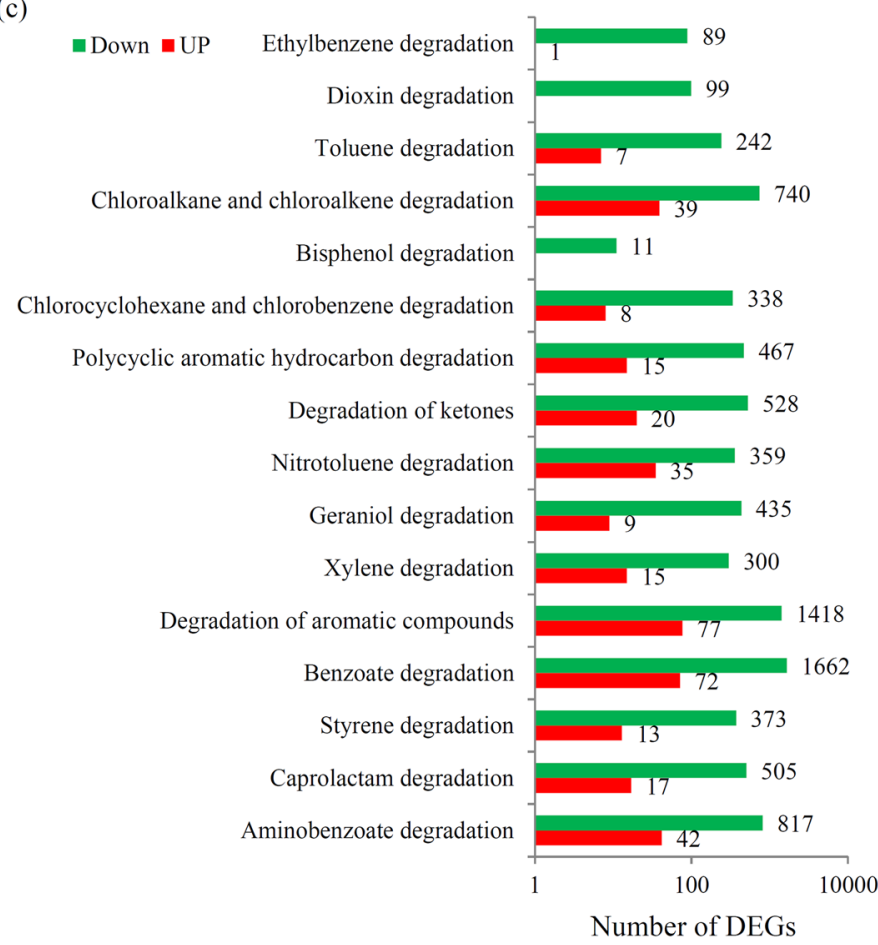

Figure 4. Analysis of differential expressed genes (DEGs). (a) The number of up- and down-regulated genes after treatment. (b) Significance analysis of the DEGs between the AT and BT sample groups by Volcanoplot. (c) The number of up- and down-regulated genes related to xenobiotic biodegradation pathways after treatment.

glu1, arcC, $C A$, and cynS. Of these genes, nirB, gltB, glu1 and cynS were up-regulated after treatment (Fig. 5a). For the sulfur metabolism pathway, 15 key genes were identified: $d s r A, c y s J, \operatorname{ssr} A, \operatorname{sir} A, f c c B, \operatorname{sre} A, \operatorname{sor} A, \operatorname{cys} K, \operatorname{met} B$, suoX, glpE, phs $A$, ssuD, ethE, and tmoC. Of these genes, sreA, sor $A, g l p E$, $p h s A$ and tmoC were up-regulated after treatment (Fig. 5b).

\section{Discussion}

Increasing evidence shows that microorganisms play an important role in biodegradation; in addition, their community structures were affected by waste gase ${ }^{28,29}$. The interaction between microorganisms and contaminants is a hot topic in research on biofiltration systems ${ }^{30}$. This study established a commercial scale biofiltration system, integrating bacterial and fungal biofilters. The study then evaluated its performance in removing odorous gases. The data showed the complete removal of $\mathrm{H}_{2} \mathrm{~S}$ and over $80 \%$ of removal for $\mathrm{NH}_{3}$. These outcomes suggest the biofiltration system was highly effective in treating odorous gases (Fig. 1). Our data help illustrate the interaction between microbial ecosystems and odorous gases removal in a biofilter at metabolic levels.

In our study, significant differences in microbial community structures were observed. Due to the high levels of $\mathrm{H}_{2} \mathrm{~S}$ and $\mathrm{NH}_{3}$, a large number of microbes could not survive in the biofilter after a week period of continuous odor-contaminated airflow. On other hand, the microbes that can utilize the ammonia and sulfite will enriched in the biofilter. Thus, the increased microbes are the target strains for improving the efficiency of biofilter.

At the phylum level, Proteobacteria was the largest phylum identified in both the BT and AT samples (Fig. 3b). Previous studies showed that several strains belonging to Proteobacteria phylum, such as Vibrio alginolyticus, Stenotrophomonas nitritireducens L2, and Nitrococcus mobilis, played important roles in removing ammonia gas ${ }^{31-33}$. Other Proteobacteria strains, such as Thiomonas sp.WZW, Pseudomonas putida, and Sulfurimonas denitrificans, played roles in removing sulfides ${ }^{15,34,35}$.

In our biofilter, the proportions of Proteobacteria increased from $38.76 \%$ to $78.99 \%$ after treatment. This indicated that Proteobacteria strains play an important role in degrading $\mathrm{H}_{2} \mathrm{~S}$ and $\mathrm{NH}_{3}$ containing gases. Further, different strains belonging to Actinobacteria and Firmicutes phyla have been identified as participating in the degradation of odorous gases ${ }^{36,37}$. For example, Rhodococcus sp. and Arthrobacter sp., belonging to Actinobacteria, were the dominant bacteria in different sulfide and ammonia treatment biofilters ${ }^{37,38}$. Many other strains belonging to Firmicutes, including Moraxella sp., Acinetobacter sp., Bacillus sp. and Exiguobacterium sp., have been found to be involved in removing ammonia and sulfide ${ }^{39,40}$. In our study, Actinobacteria and Firmicutes were the top 10 largest phyla after the treatment, suggesting they play roles in degrading odorous gases. Our data provided important cues for isolation of dominant microbes in biofilter after odorous gas treatment.

A previous study found that Acidithiobacillus thiooxidans AZ11 is a sulfate-resistant strain and exhibits high sulfur oxidizing activity at low $\mathrm{pH}$ conditions ${ }^{16}$. At the genera level, Acidithiobacillus (70.1\%) was the dominant genus after odorous gas treatment (Table S3). In addition to Acidithiobacillus, Thiomonas sp. WZW has been enriched and isolated from activated sewage sludge and has been shown to play an important role in enhancing 


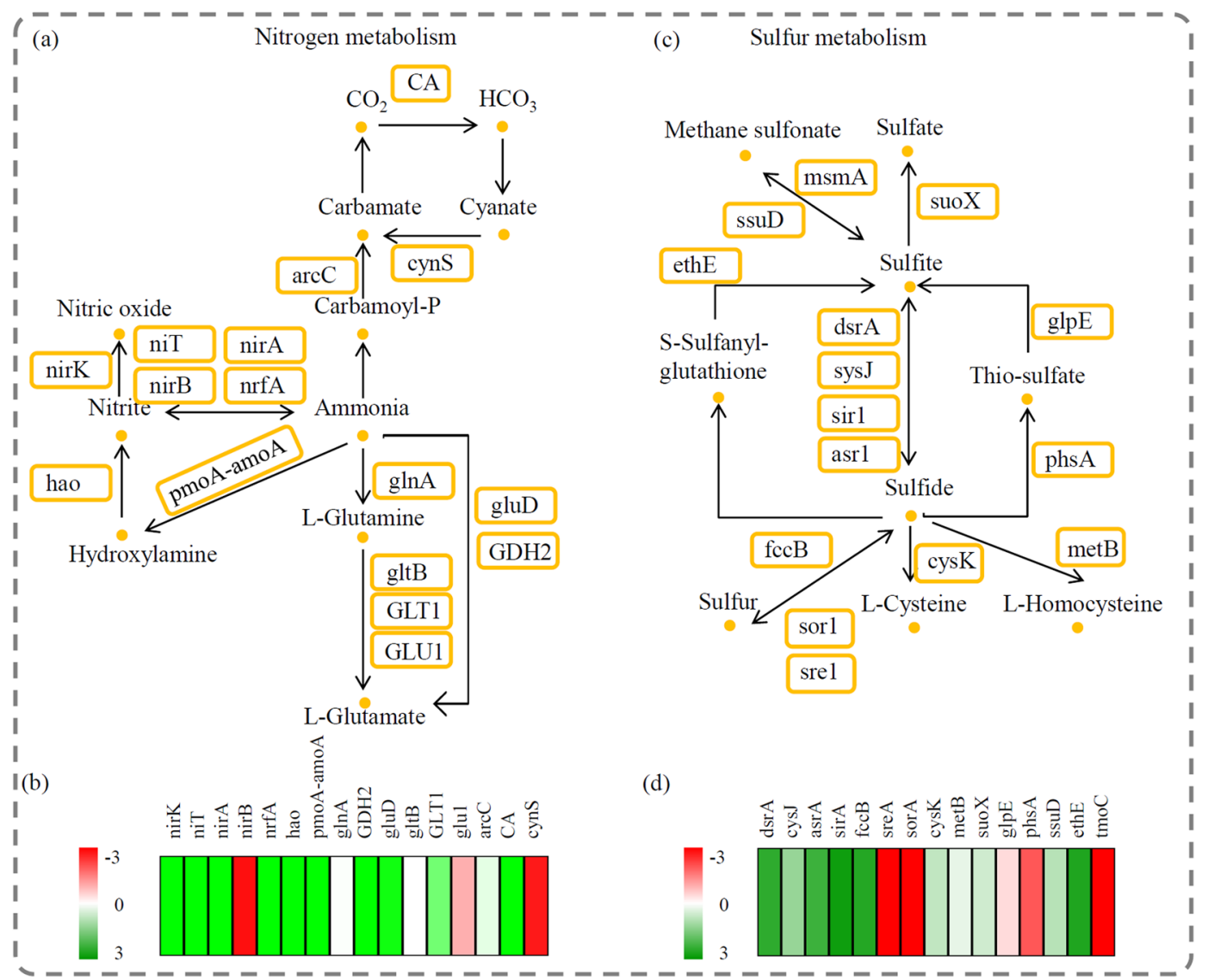

Figure 5. Analysis of the genes involved in the nitrogen metabolic and sulfur metabolic pathways. (a) Overview of the nitrogen metabolic pathway. (b) The relative abundances of the genes involved in the nitrogen metabolic pathway. (b) Overview of the sulfur metabolic pathway. (b) The relative abundances of the genes involved in the sulfur metabolic pathway. Red indicated up-regulated and green indicated down-regulated genes. The heatmap scale ranges from -3 to +3 on a $\log _{2}$ scale.

the removal of carbon disulfide ${ }^{15}$. In this study, the genus Thiomonas significantly increased (from $0.073 \%$ to $0.43 \%$ ) after treating odorous gases. Based on the high effective removal of $\mathrm{NH}_{3}$ in our biofilter, strains belonging to Acidithiobacillus and Thiomonas genera may play roles in removing ammonia.

$\mathrm{NH}_{3}$ and $\mathrm{H}_{2} \mathrm{~S}$ are two important indicators for the removal of odorous gases ${ }^{41,42}$. In the past decade, biofilters with different packing media have been widely used to simultaneously remove $\mathrm{NH}_{3}$ and $\mathrm{H}_{2} \mathrm{~S}$ gases ${ }^{43}$. In our study, most of the genes involved in ammonia and sulfite metabolism were identified in the BT group, and not the AT group. This is consistent with the reduction in microbial community structures in the BT group, compared with the AT group (Fig. 5). A large number of microbes without the capacity in absorption and fixation of $\mathrm{NH}_{3}$ and $\mathrm{H}_{2} \mathrm{~S}$ could not survive in the biofilter.

Due to the reduction in microbial community structures, the relative abundance of many ammonia metabolism-related genes was decreased in the BT group. Ammonium ion assimilation involves a central metabolic pathway in various plants; glutamine synthetase plays an essential role of incorporating ammonium ions into glutamine and glutamate ${ }^{44}$. Based on different cofactors, glutamine synthetase occurs in three forms: gltB (EC:1.4.1.13, using NADPH), glt1 (EC: 1.4.1.13, using NADH), and glu1 (EC:1.4.7.1, using reduced ferredoxin) ${ }^{45}$. This study identified all three glutamine synthetase forms. There was an increase in the relative abundances of gltB (EC: 1.4.1.13) and glu1 (EC:1.4.7.1). This suggests that gltB and glu1 were the dominant forms of glutamine synthetase when treating odorous gases. NirB is essential for NADH-dependent nitrite reductase activity ${ }^{46}$. The relative abundance of nirB (EC: 1.7.1.15, K00362) was higher in the AT group; this suggests it plays a role in assimilating ammonia-containing odorous gases.

For the sulfur metabolism pathway, five key gene categories were highly expressed in the AT sample group. SreA is an important gene encoding $110 \mathrm{kDa}$ subunit of sulfur oxygenase/reductase; and sorA is another gene encoding 309 amino acid residues of sulfur oxygenase/reductase $e^{47,48}$. In this study, there was a higher relative abundance of both sreA and sorA genes in the AT group compared to the BT group. This suggests there was an activated sulfur oxidation pathway in the biofilter. In addition, $g l p E$, encoding a thiosulfate/3-mercaptopyruvate sulfurtransferase; $p h s A$, encoding a thiosulfate reductase; and $e t h E$, encoding a sulfur dioxygenase, were significantly induced by the odorous gas treatment ${ }^{49,50}$. Sre $1 A$, sor $A$, phs $A$, and phsA were involved in sulfur metabolism and were considered to be efficient genes responsible for sulfur fixation in our biofilter. 
The performance of biofilters in removing odorous gases was determined based on microbial compositions and metabolic interactions. Based on unigene sequences, significantly differences in microbial community structures were seen between the BT and AT sample groups. At the phylum level, proportions of Proteobacteria, Euryarchaeota, Nitrospirae, and Parvarchaeota significantly increased after treatment, indicating their important roles in degrading gases containing $\mathrm{H}_{2} \mathrm{~S}$ and $\mathrm{NH}_{3}$. Several target strains will be used for improving the efficiency of biofilter by artificial addition. Most genes involved in ammonia and sulfite metabolisms were in the BT group, rather than the AT group. An analysis of the relative abundance identified several key up-regulated genes, which may be the most efficient genes involved in removing odorous gases. Furthermore, efficient genes involved in removing odorous gases will be used for the genetic improvement of target strains. Our findings can be used for improving the efficiency of biofilter and helping the industrial enterprises to reduce the emission of waste gas.

Received: 26 June 2019; Accepted: 7 November 2019;

Published online: 28 November 2019

\section{References}

1. Wu, C. et al. Microbial compositions and metabolic interactions in one- and two-phase partitioning airlift bioreactors treating a complex VOC mixture. J Ind Microbiol Biotechnol 44, 1313-1324 (2017).

2. Balasubramanian, P., Philip, L. \& Murty Bhallamudi, S. Biotrickling filtration of complex pharmaceutical VOC emissions along with chloroform. Bioresour Technol 114, 149-159 (2012).

3. Lim, J. H., Cha, J. S., Kong, B. J. \& Baek, S. H. Characterization of odorous gases at landfill site and in surrounding areas. Journal of Environmental Management 206, 291-303 (2018).

4. Yuan, H., Du, X., Tai, H. \& Xu, M. Temperature-programmed multicapillary gas chromatograph microcolumn for the analysis of odorous sulfur pollutants. J Sep Sci 41, 893-898 (2018).

5. Wu, H. et al. Recent progress and perspectives in biotrickling filters for VOCs and odorous gases treatment. J Environ Manage 222, 409-419 (2018)

6. Wan, S., Li, G., An, T. \& Guo, B. Co-treatment of single, binary and ternary mixture gas of ethanethiol, dimethyl disulfide and thioanisole in a biotrickling filter seeded with Lysinibacillus sphaericus RG-1. J Hazard Mater 186, 1050-1057 (2011).

7. Ghoshal, A. K. \& Manjare, S. D. Selection of appropriate adsorption technique for recovery of VOCs: an analysis. Journal of Loss Prevention in the Process Industries 15, 413-421 (2002).

8. Ryu, H. W., Cho, K. S. \& Lee, T. H. Reduction of ammonia and volatile organic compounds from food waste-composting facilities using a novel anti-clogging biofilter system. Bioresour Technol 102, 4654-4660 (2011).

9. Gaudin, F., Andres, Y. \& Le Cloirec, P. Packing material formulation for odorous emission biofiltration. Chemosphere 70, 958-966 (2008).

10. Revah, S. \& Morgan-Sagastume, J. M. Methods of Odor and VOC Control (2005).

11. Bayle, S., Malhautier, L., Degrange, V., Godon, J. J. \& Fanlo, J. L. Structural and functional responses of sewage microbial communities used for the treatment of a complex mixture of volatile organic compounds (VOCs). J Appl Microbiol 107, 85-96 (2009).

12. Sundberg, C. et al. Effects of $\mathrm{pH}$ and microbial composition on odour in food waste composting. Waste Manag 33, 204-211 (2013).

13. Ralebitso-Senior, T. K., Senior, E., Di Felice, R. \& Jarvis, K. Waste gas biofiltration: advances and limitations of current approaches in microbiology. Environ Sci Technol 46, 8542-8573 (2012).

14. Eulsaeng, C., Galera, M. M., Lorenzana, A. \& Wookjin, C. Ethylbenzene, o-xylene, and BTEX removal by Sphingomonas sp. D3K1 in rock wool-compost biofilters. Environmental Engineering Science 26, 45-52 (2009).

15. Wani, A. H., Lau, A. K. \& Branion, R. M. Biofiltration control of pulping odors - hydrogen sulfide: performance, macrokinetics and coexistence effects of organo-sulfur species. Journal of Chemical Technology \& Biotechnology 74, 9-16 (1999).

16. Lee, E. Y., Lee, N. Y., Cho, K. S. \& Ryu, H. W. Removal of hydrogen sulfide by sulfate-resistant Acidithiobacillus thiooxidans AZ11. J Biosci Bioeng 101, 309-314 (2006).

17. Ye, L., Zhang, T., Wang, T. \& Fang, Z. Microbial Structures, Functions, and Metabolic Pathways in Wastewater Treatment Bioreactors Revealed Using High-Throughput Sequencing. Environmental Science \& Technology 46, 13244-13252 (2012).

18. Abia, A. L. K., Alisoltani, A., Ubomba-Jaswa, E. \& Dippenaar, M. A. Microbial life beyond the grave: $16 \mathrm{~S}$ rRNA gene-based metagenomic analysis of bacteria diversity and their functional profiles in cemetery environments. Sci Total Environ 655, 831-841 (2018).

19. Bai, Y., Huo, Y., Liao, K. \& Qu, J. Influence of microbial community diversity and function on pollutant removal in ecological wastewater treatment. Appl Microbiol Biotechnol 101, 7293-7302 (2017).

20. Ueno, H., Amano, S., Merecka, B. \& Kosmider, J. Difference in the odor concentrations measured by the triangle odor bag method and dynamic olfactometry. Water Sci Technol 59, 1339-1342 (2009).

21. Noguchi, M., Tanaka, S., Watanabe, K. \& Yamasaki, A. Correlation between Odor Concentration and Volatile Organic Compounds (VOC) Composition of Environmental Tobacco Smoke (ETS). International journal of environmental research and public health 13, 994 (2016).

22. Kolacinska, K. \& Koncki, R. A novel optoelectronic detector and improved flow analysis procedure for ammonia determination with Nessler's reagent. Anal Sci 30, 1019-1022 (2014).

23. Cheng, M. et al. A metagenomics approach to the intestinal microbiome structure and function in high fat diet-induced obesity mice fed with oolong tea polyphenols. Food Funct 9, 1079-1087 (2018).

24. He, F. et al. Metagenomic analysis of captive Amur tiger faecal microbiome. BMC Vet Res 14, 379 (2018).

25. Chao, A. \& Shen, T. J. Nonparametric estimation of Shannon's index of diversity when there are unseen species in sample. Environmental \& Ecological Statistics 10, 429-443 (2003).

26. Green, G. H. \& Diggle, P. J. On the operational characteristics of the Benjamini and Hochberg False Discovery Rate procedure. Stat Appl Genet Mol Biol 6, Article27 (2007).

27. Statheropoulos, M., Agapiou, A., Spiliopoulou, C., Pallis, G. C. \& Sianos, E. Environmental aspects of VOCs evolved in the early stages of human decomposition. Sci Total Environ 385, 221-227 (2007).

28. Lu, L., Wang, G., Yeung, M., Xi, J. \& Hu, H.-Y. Response of microbial community structure and metabolic profile to shifts of inlet VOCs in a gas-phase biofilter. AMB Express 8, 160-160 (2018).

29. Qiu, G., Song, Y., Zeng, P., Duan, L. \& Xiao, S. Combination of upflow anaerobic sludge blanket (UASB) and membrane bioreactor (MBR) for berberine reduction from wastewater and the effects of berberine on bacterial community dynamics. J Hazard Mater 246-247, 34-43 (2013).

30. Obi, C. C. et al. Structural dynamics of microbial communities in polycyclic aromatic hydrocarbon-contaminated tropical estuarine sediments undergoing simulated aerobic biotreatment. Appl Microbiol Biotechnol 101, 4299-4314 (2017).

31. Kim, N.-J., Sugano, Y., Hirai, M. \& Shoda, M. Removal of a high load of ammonia gas by a marine bacterium, Vibrio alginolyticus. Journal of Bioscience and Bioengineering 90, 410-415 (2000). 
32. Finkmann, W., Altendorf, K., Stackebrandt, E. \& Lipski, A. Characterization of N2O-producing Xanthomonas-like isolates from biofilters as Stenotrophomonas nitritireducens sp. nov., Luteimonas mephitis gen. nov., sp. nov. and Pseudoxanthomonas broegbernensis gen. nov., sp. nov. International Journal of Systematic and Evolutionary Microbiology 50, 273-282 (2000).

33. Juhler, S. et al. Distribution and rate of microbial processes in an ammonia-loaded air filter biofilm. Appl Environ Microbiol 75, 3705-3713 (2009).

34. Zilli, M., Converti, A. \& Di Felice, R. Macrokinetic and quantitative microbial investigation on a bench-scale biofilter treating styrene-polluted gaseous streams. Biotechnol Bioeng 83, 29-38 (2003).

35. Maestre, J. P. et al. Bacterial community analysis of a gas-phase biotrickling filter for biogas mimics desulfurization through the rRNA approach. Chemosphere 80, 872-880 (2010).

36. Aldric, J. M. \& Thonart, P. Performance evaluation of a water/silicone oil two-phase partitioning bioreactor using Rhodococcus erythropolis T902.1 to remove volatile organic compounds from gaseous effluents. Journal of Chemical Technology \& Biotechnology 83, 1401-1408 (2010).

37. Anja, K. et al. Butyric acid- and dimethyl disulfide-assimilating microorganisms in a biofilter treating air emissions from a livestock facility. Applied \& Environmental Microbiology 77, 8595 (2011).

38. Chung, Y. C., Liu, C. H. \& Huang, C. Feasibility of fluidized-bed bioreactor for remediating waste gas containing H2S or NH3. J Environ Sci Health A Tox Hazard Subst Environ Eng 36, 509-520 (2001).

39. Xie, B., Liang, S. B., Tang, Y., Mi, W. X. \& Xu, Y. Petrochemical wastewater odor treatment by biofiltration. Bioresour Technol 100, 2204-2209 (2009).

40. Omri, I., Bouallagui, H., Aouidi, F., Godon, J. J. \& Hamdi, M. H2S gas biological removal efficiency and bacterial community diversity in biofilter treating wastewater odor. Bioresour Technol 102, 10202-10209 (2011).

41. Subrenat, A., Le Leuch, L. M. \& Le Cloirec, P. Electrodeposition of copper and iron oxides on to activated carbon fibre cloths: application to H2S and NH3 removal from air. Environ Technol 29, 993-1000 (2008).

42. Jiang, X. \& Tay, J. H. Operational characteristics of efficient co-removal of $\mathrm{H} 2 \mathrm{~S}$ and $\mathrm{NH} 3$ in a horizontal biotrickling filter using exhausted carbon. Journal of Hazardous Materials 176, 638-643 (2010).

43. Hou, J. et al. Simultaneous removal of ammonia and hydrogen sulfide gases using biofilter media from the biodehydration stage and curing stage of composting. Environ Sci Pollut Res Int 23, 20628-20636 (2016)

44. van den Heuvel, R. H., Curti, B., Vanoni, M. A. \& Mattevi, A. Glutamate synthase: a fascinating pathway from L-glutamine to L-glutamate. Cell Mol Life Sci 61, 669-681 (2004).

45. Suzuki, A. \& Knaff, D. B. Glutamate synthase: structural, mechanistic and regulatory properties, and role in the amino acid metabolism. Photosynth Res 83, 191-217 (2005).

46. Harborne, N. R., Griffiths, L., Busby, S. J. \& Cole, J. A. Transcriptional control, translation and function of the products of the five open reading frames of the Escherichia coli nir operon. Mol Microbiol 6, 2805-2813 (1992).

47. Laska, S., Lottspeich, F. \& Kletzin, A. Membrane-bound hydrogenase and sulfur reductase of the hyperthermophilic and acidophilic archaeon Acidianus ambivalens. Microbiology 149, 2357-2371 (2003).

48. Urich, T. et al. Identification of core active site residues of the sulfur oxygenase reductase from Acidianus ambivalens by site-directed mutagenesis. FEMS Microbiol Lett 248, 171-176 (2005).

49. Hinsley, A. P. \& Berks, B. C. Specificity of respiratory pathways involved in the reduction of sulfur compounds by Salmonella enterica. Microbiology 148, 3631-3638 (2002).

50. Kabil, O. \& Banerjee, R. Characterization of patient mutations in human persulfide dioxygenase (ETHE1) involved in H2S catabolism. J Biol Chem 287, 44561-44567 (2012).

\section{Acknowledgements}

We are also grateful to LC Sciences company (Hangzhou, China) for technical support. This work was funded by the National Natural Science Foundation of China (Grant No. 21207028), Zhejiang Provincial Natural Science Foundation of China (Grant No. LY15B070013), and Hangzhou Science and Technology Development Foundation of China (Grant No. 20190101A02).

\section{Author contributions}

Conceptualization: Weidong Li and Qi Liu; Data curation, Weidong Li, Shaoqin Cai, Jianguo Ni, and Ying Liu; Formal analysis: Chenjia Shen and Huayun Yang; Funding acquisition, Qi Liu; Investigation: Weidong Li, Shaoqin Cai, Jianguo Ni, Jia Tao, Yunfeng Yu, and Ying Liu; Methodology: Jianguo Ni; Resources: Weidong Li, Huayun Yang, and Yuquan Chen; Software: Chenjia Shen; Writing - original draft: Chenjia Shen, and Qi Liu.

\section{Competing interests}

The authors declare no competing interests.

\section{Additional information}

Supplementary information is available for this paper at https://doi.org/10.1038/s41598-019-54281-0.

Correspondence and requests for materials should be addressed to Q.L.

Reprints and permissions information is available at www.nature.com/reprints.

Publisher's note Springer Nature remains neutral with regard to jurisdictional claims in published maps and institutional affiliations.

Open Access This article is licensed under a Creative Commons Attribution 4.0 International License, which permits use, sharing, adaptation, distribution and reproduction in any medium or format, as long as you give appropriate credit to the original author(s) and the source, provide a link to the Creative Commons license, and indicate if changes were made. The images or other third party material in this article are included in the article's Creative Commons license, unless indicated otherwise in a credit line to the material. If material is not included in the article's Creative Commons license and your intended use is not permitted by statutory regulation or exceeds the permitted use, you will need to obtain permission directly from the copyright holder. To view a copy of this license, visit http://creativecommons.org/licenses/by/4.0/.

(C) The Author(s) 2019 